E-Review Dossier 1-2013

Bologna (BraDypUS) 2014

La crisi dei partiti in Emilia

Romagna negli anni'70/'80

a cura di Mirco Carrattieri

e Carlo De Maria

ISSN: 2282-4979

ISBN: $978-88-98392-05-6$

D0I: 10.12977/ereview22

pp. 21-54

Questo articolo è stato sottoposto a un processo di double blind peer review

\title{
dell'Emilia Romagna.
}

Lineamenti di un dibattito

comparato

L'impostazione antiburocratica del regionalismo dei primi anni Settanta puntò sullo sviluppo della programmazione. Una declinazione peculiare di questo metodo di governo si affermò in Emilia Romagna. Dopo il 1976 intervenne una svolta centralistica a livello nazionale, che snaturò il ruolo costituzionale delle regioni. Nel decennio successivo, mentre la Regione Emilia Romagna provava a lanciare una nuova fase costituente, si assistette in Italia al declino dell'idea stessa di programmazione.

In the early seventies the anti-bureaucratic setting of the regionalism aimed on the development of regional planning. A peculiar variation of this form of government established itself in Emilia Romagna. After 1976, a centralistic turning intervened at the national level, which denaturalised the constitutional role of the regions. Over the next decade, while the Emilia Romagna tried to launch a new constituent phase, we witnessed in Italy to the decline of the idea of planning.

\section{Introduzione}

L'attuazione dell'ordinamento regionale, nel 1970, non fu un semplice residuato della Costituzione, o una concessione distratta a una pressione che veniva da sinistra. Il rilancio del regionalismo fu piuttosto connesso all'inasprirsi di antichi e nuovi squilibri, conseguenza dei meccanismi di sviluppo innescatisi negli anni Cinquanta e Sessanta [Ridolfi 2010, 124]. Si stavano determinando nel paese veri e propri processi di disgregazione, in rapporto sia all'assetto del territorio che alla carenza di servizi sociali: «problemi che non potevano più essere affrontati né a 
livello municipale, né con gli schemi del vecchio Stato centralizzato e ministeriale» [Ingrao 1973, 2].

La riforma regionale tentò di ricomporre una diaspora territoriale che frantumava la penisola in una miriade di piattaforme localistiche; una situazione che avrebbe annullato ogni possibilità di articolare e programmare efficacemente l'intervento pubblico. Per la prima volta dal 1947-48 l'arco delle forze regionaliste travalicò in modo consistente i confini della sinistra socialista e comunista, coinvolse gruppi importanti della maggioranza governativa di centro-sinistra e diede una base di sostegno nuova, più larga, alla ricerca di una organizzazione diversa della macchina statale.

Nello stesso tempo, l'introduzione delle regioni a statuto ordinario mise in discussione il tradizionale modo di essere dei partiti e la loro capacità di esprimere una sintesi politica generale o nazionale. La posta in gioco era chiara fin dall'inizio degli anni Sessanta: una piena attuazione del regionalismo avrebbe presupposto una ristrutturazione della forma partito all'insegna di una maggiore autonomia delle espressioni territoriali. Il Partito comunista italiano se ne dimostrò consapevole già durante i lavori del IX congresso (1960), dove si mise in rilievo la necessità di porre mano a organi regionali di direzione e di iniziativa politica. Nel definire i compiti di questi nuovi «comitati regionali di coordinamento», le risoluzioni congressuali facevano riferimento a «centri di direzione regionale» in grado di assicurare «il superamento dei limiti delle federazioni [provinciali]», articolando a livello delle regioni «la generale politica di rinnovamento strutturale»; ciò sarebbe potuto avvenire solo sulla base di una «aggiornata conoscenza della realtà regionale e di una capacità di iniziativa a questo livello».

Le spinte politiche più significative in questa direzione provennero dall'Emilia Romagna. Un primato nazionale esplicitamente rivendicato dal segretario regionale, il ravennate Sergio Cavina, che alla vigilia del 1970 indicava al proprio partito la «necessità di una verifica critica, politico-organizzativa», sul tema del decentramento interno, affermando altresì che l'Emilia Romagna avrebbe dovuto fare «più a fondo questa verifica, perché noi siamo l'organizzazione che più ha sperimentato in questo campo»².

Pci, Direzione nazionale, Risoluzione sui comitati regionali, documento interno, 16 marzo 1960, in Fondazione Gramsci Emilia Romagna (d'ora in poi FGER), Archivio del Comitato regionale comunista (PCI CR-ER), Verbali e risoluzioni, b.1, f.1960-1963.

PCi, Comitato regionale Emilia Romagna, riunione del 12 ottobre 1968, verbale ms., in FGER, PCI CR-ER, Segreteria, b.1, f. 1968 . 
Occorreva accentuare il ruolo delle sezioni locali e dei comitati regionali, al posto del vecchio rapporto gerarchico tra la direzione nazionale e le federazioni provinciali. Ma questo avvenne solo in piccola misura, se è vero che, analizzando quindici anni più tardi il gruppo dirigente nazionale del Pci, Farneti osservò come il passo più importante nella carriera dei dirigenti comunisti rimanesse il livello della federazione provinciale: solo lentamente le cariche di membro o di segretario del comitato regionale andarono assumendo maggiore importanza nel cursus honorum dei dirigenti del partito [Farneti 1983].

Non erano mancati momenti di tensione tra Bologna e Roma. Basti ricordare che nel 1962 il comitato regionale emiliano-romagnolo aveva approvato una proposta di riforma organizzativa del partito con potenzialità dirompenti ${ }^{3}$. Si prefigurava una «rifondazione democratica» del Pci basata su centri di direzione politica autonomi: «un regionalismo spinto ai limiti, si direbbe oggi, di un partito federativo» [Fanti 2001, 113]. La proposta proveniente da Bologna circolò brevemente nel dibattito nazionale fino alla conclusione del $\mathrm{X}$ congresso, quando risultò nettamente perdente di fronte alle resistenze centralistiche dominanti nella direzione del partito.

Nonostante i buoni propositi espressi nel 1960, mancava ai vertici del Pci, come ai gruppi dirigenti delle altre formazioni politiche, una autentica visione regionalista. Del resto, sarebbe stato sorprendente il contrario. Per decenni, infatti, l'organizzazione gerarchica dei partiti si era perfettamente sovrapposta - in maniera quasi naturale - al tradizionale centralismo amministrativo dello Stato unitario [Tarrow 1979a; Cassese 1986]. Si ebbe una conferma di questa continuità lungo gli anni Settanta e Ottanta, quando nei consigli regionali emerse una classe politica nuova, in larga parte proveniente da esperienze nelle amministrazioni locali; e tuttavia tale rinnovamento trovò un suo limite sostanziale nella condotta dei partiti, che tesero a vanificare gli spazi di autonoma iniziativa politica continuando a privilegiare un controllo verticistico sulle periferie [Scoppola 2003].

Le forze politiche non vollero o non seppero cogliere la possibilità di un vero $\mathrm{e}$ proprio salto di qualità che era insito nella istituzione delle regioni a statuto ordinario. Secondo Lanfranco Turci, terzo presidente della regione Emilia Romagna (1978-87), si preferì interpretare l'ente regione come «una generica estensione delle tradizionali esperienze di amministrazione locale». Non si comprese la sua portata come vero e proprio organo di governo e come soggetto legislativo:

Pci, Comitato regionale Emilia Romagna, Note su alcuni problemi organizzativi del partito in Emilia Romagna, 1962, in FGER, PCI CR-ER, Verbali e risoluzioni, b.1, f.1960-1963. 
da parte nostra in particolare [il riferimento è al Pci] non si è concentrato né uno sforzo programmatico né un ripensamento istituzionale adeguati su questo atto importante, sia pur tardivo, di attuazione delle Costituzione. Ne è derivato conseguentemente un isolamento delle importanti esperienze di governo messe in atto nelle regioni "rosse", esperienze che non sono state riprese né concettualizzate per il loro possibile significato nazionale.

Nella lettura critica di Turci il prevalere di una visione tradizionale e continuistica sui temi delle autonomie tenne imbrigliato il Pci «in quel mix di difensivismo e rivendicazionismo municipalistico» che costituiva il limite culturale di tanto autonomismo e che aveva nell'Anci la sua espressione più emblematica: «si potrebbe dire che c'è una vera e propria cultura dell'Anci che ispira molta parte del nostro Partito e dei gruppi parlamentari, producendo guasti minori ma significativi»4. Questi ritardi sono confermati dalla consultazione delle carte del comitato regionale comunista (presso la Fondazione Gramsci Emilia Romagna) e dallo spoglio di una rivista di area Pci come "Regione e governo locale" (edita a Bologna dal 1980 e trasformatasi nel 1997 in "Le istituzioni del federalismo"), sedi importanti di confronto politico e dibattitto scientifico dove, fino a tutti gli anni Ottanta, non venne mai pronunciata la parola "federalismo", che sembrava costituire un tabù. La regione, cioè, veniva perlopiù vista come una mera proiezione dell'autonomia comunale, quando invece avrebbe potuto chiamare in causa una riflessione politico-culturale sul principio federale. Il dibattito interno alla sinistra continuò sostanzialmente a vertere sul "decentramento", si limitò al piano amministrativo, piuttosto che coinvolgere quello propriamente politico, della critica allo statalismo.

Si tratta di elementi poco frequentati dal dibattito storiografico. Infatti, la letteratura sull'attuazione e sul consolidamento delle regioni a statuto ordinario è stata ampia fin dall'inizio, ma improntata in prevalenza all'indagine giuridica. Un peso minore ha avuto, almeno nei primi due decenni, la ricerca politologica, sia sul versante istituzionale - l'introduzione di un nuovo livello di governo nel sistema politico -, che sul piano dei rapporti con la società: una nuova arena per i processi di espressione della domanda, di transazione e decisione [Passigli 1987]. Gli storici contemporaneisti hanno cominciato a muoversi su questo tema solamente negli anni Duemila. È stato Roberto Balzani [2004] a definire il periodo 1970-90 come il «ventennio aurorale e quasi archeologico» dell'ente regione. La discontinuità che si registra, rispetto al panorama odierno, nella cultura politica e nei

L. Turci, appunti in preparazione di un articolo per "Rinascita", 28 dicembre 1985, in Archivio storico della Regione Emilia Romagna (d'ora in poi ASRER), Presidenza della Giunta, Segreteria del Presidente, Sezione Presidenza Turci, b.831, f. "Interventi, articoli ecc. presidente Turci". 
linguaggi - si pensi al declino politico e culturale dell'idea stessa di programmazione - può ormai consentire, secondo lo storico romagnolo, una ricerca pienamente scientifica sulle fonti primarie. Va, cioè, aperta la fase della ricerca d'archivio.

Il termine ad quem individuato da Balzani, il 1990, è significativo anche dal punto di vista degli statuti regionali. Quello dell'Emilia Romagna veniva modificato proprio allora, ma più in generale si ritoccarono in quel periodo anche gli equilibri normativi degli altri statuti ordinari. Mentre all'inizio degli anni Settanta si era optato generalmente per una forma di governo a tendenza assembleare sul modello della Costituzione repubblicana - riproducendo, cioè, a livello regionale la "democrazia dei partiti" fissata sulla carta fondamentale -, nell'esperienza successiva le leggi regionali intesero modificare i rapporti tra gli organi a favore dell'esecutivo [Vandelli 1990].

\section{La programmazione regionale: ipotesi o realtà?}

Dopo che la prima legislatura era stata in buona parte assorbita dall'elaborazione delle "regole", la seconda avviò - in seguito alla legislazione del 1975-77 - la fase più alta del tentativo di programmazione. Si tratta di una storia ancora tutta da scrivere nella quale un ruolo di rilievo spetta ai programmi regionali di sviluppo, che a partire dal 1978 puntarono a rafforzare un concreto potere di coordinamento e di pianificazione del nuovo ente.

Per la verità, l'ipotesi della programmazione caratterizzò sin dalla fase iniziale l'esperienza delle regioni. Molti statuti la recepirono, seppure in forma generica, e alcune esperienze di governo - come si vedrà nel prossimo paragrafo - vi si confrontarono già nella prima legislatura.

Ma fu solo in seguito alla legge 335 del 1976, sulla contabilità regionale, che divenne possibile adottare, accanto al bilancio annuale, un bilancio pluriennale. Se il primo continuava a svolgere la funzione di documento contabile essenziale per registrare l'andamento delle entrate e delle uscite, con il secondo si potevano fissare obiettivi di medio periodo e quindi definire politiche pubbliche e di sviluppo con i relativi impegni di spesa.

L'anno successivo il decreto presidenziale 616, attuativo della legge 382/1975 sul trasferimento dei poteri alle regioni, definì più compiutamente i contenuti e gli strumenti della programmazione. Contemporaneamente, però, iniziò uno stillicidio di provvedimenti settoriali, nei campi trasferiti alle regioni in modo più o meno completo (lavori pubblici, agricoltura, artigianato, assistenza sociale), alimentando una frammentarietà che incise subito negativamente sulla possibilità di sviluppo delle funzioni programmatorie. 
Speranze, incertezze e delusioni alimentarono una vastissima letteratura in tema di programmazione regionale, accompagnando almeno i primi 10-15 anni di storia delle regioni. In quel periodo i due termini "regione" e "programmazione" vennero tante volte affiancati da divenire, almeno sulla carta, un binomio indissolubile. E tuttavia non si arrivò a una reale e definitiva chiarificazione su che cosa fosse la programmazione regionale.

Ciò che sembra certo è che si produsse una sostanziale revisione di quella che veniva spesso chiamata la "filosofia illuministica" della programmazione. Tipica degli anni Sessanta, tale impostazione si era fondata sull'ottimistica fiducia che lo Stato potesse comporre in un quadro razionale lo sviluppo complessivo del sistema economico. Essa, già all'inizio degli anni Settanta, apparteneva ormai alla storia della programmazione e tutta l'esperienza delle regioni, dalla fase "costituente" in poi, mosse da una concezione diversa, sintetizzata da una formula assai ricorrente nel discorso pubblico di quegli anni: "la programmazione come metodo di governo". Un approccio che si concretizzava non tanto nell'elaborazione di un singolo atto formale, il piano, quanto piuttosto nel modo di articolarsi dell'intera attività dell'ente impegnato nella programmazione: una organizzazione meditata dei processi decisionali diretta a conseguire una coerenza dell'indirizzo politico nel suo complesso.

In ogni caso, a questo mutamento di concezione corrispose generalmente una ben misera prassi programmatoria da parte delle regioni. I motivi del fallimento, all'inizio degli anni Ottanta, erano stati identificati con una certa precisione dagli studi giuridici: l'assetto frastagliato delle competenze; l'impossibilità di definire una manovra di bilancio all'interno di un quadro di finanza derivata composta da assegnazioni statali vincolate; l'assenza di una programmazione nazionale con la quale interagire; i ritardi nella riforma complessiva delle autonomie.

Secondo Roberto Bin [1982] anche l'importanza del decreto 616 fu troppo enfatizzata, se non altro per quel che riguardava le sue concrete implicazioni sulla possibilità di varare una efficiente programmazione regionale. Tanto per cominciare, era troppo scarsa l'omogeneità delle funzioni trasferite dal decreto presidenziale. In secondo luogo, l'effetto positivo del 616 dipendeva strettamente dalla questione delle leggi cornice, che spesso mancavano (lasciando incertezza nella delimitazione delle competenze), e laddove erano state emanate, operavano quasi sempre un restringimento dell'autonomia regionale, introducendo norme di dettaglio, spesso minutissime, anziché norme di principio.

Un effetto deleterio produssero poi le leggi statali di programmazione settoriale (si pensi, ad esempio, alla cosiddetta "legge quadrifoglio" n. 984/77, relativa al coordinamento degli interventi pubblici in quattro settori: quelli della zootecnia, 
delle forestazione, della produzione ortofrutticola e vitivinicola) almeno per due ragioni: esse crearono collegamenti a canna d'organo tra Stato e regioni, introducendo procedimenti e strumenti diversi da settore a settore (proprio il contrario del concetto di programmazione regionale), e ridussero ulteriormente l'autonomia finanziaria della regione vincolando ancora di più le assegnazioni statali.

Il fattore di maggior stimolo e novità per la programmazione regionale rimase indubbiamente la riforma della contabilità realizzata con la già citata legge 335/76, che introdusse la possibilità del bilancio pluriennale come proiezione finanziaria dei piani regionali di sviluppo. Sicuramente una svolta nella storia amministrativa italiana, ma impotente di fronte al problema di fondo della finanza regionale, sempre più vincolata dall'alto.

Come se ciò non bastasse, sullo scorcio di quel decennio si manifestò anche la "crisi ideologica" della innovazione istituzionale che maggiormente aveva caratterizzato la fase costituente delle regioni ordinarie, ovverosia la nascita dei comprensori, a cui era legata l'impostazione antiburocratica del regionalismo dei primi anni Settanta (su questo punto si rinvia al par. 6).

\section{L'Emilia Romagna e la "programmazione democratica"}

Una declinazione peculiare dell'idea di programmazione si era affermata negli enti locali emiliani, e in particolare a Bologna, fin dagli anni Sessanta, quando si dispiegò quella che è stata definita la «funzione nazionale del modello emiliano» [Cammelli 1978].

Si trattò di una linea di "anticipazioni” tesa a precorrere e, contemporaneamente, a influenzare le riforme nazionali con l'obiettivo di favorire la legittimazione del Pci al governo del paese e di rompere l'isolamento nel quale erano tenuti i comunisti rispetto all'esperienza di centro-sinistra. Anticipazioni che si basarono sull'uso innovativo di tradizionali strumenti amministrativi e sull'invenzione di nuove soluzioni istituzionali, come accadde con i consigli di quartiere a Bologna. Questa stagione si sarebbe estesa anche alla prima fase della esperienza regionale, connotandone significativamente la stessa attività legislativa, con riferimento ai provvedimenti regionali che nella prima legislatura (1970-75) prefigurarono nuove articolazioni istituzionali generali (comitati comprensoriali) o di settore (consorzi socio-sanitari). Sia le une che le altre nacquero da forme volontarie di associazionismo fra comuni, che resero possibile l'avvio di un processo di programmazione sul territorio.

Se è vero che la programmazione, tra anni Sessanta e Settanta, era un tema caldo anche nel dibattito pubblico nazionale (si pensi al varo da parte del governo 
del controverso Programma di sviluppo economico per il 1966-70), tuttavia in Emilia Romagna essa mostrava una caratteristica curvatura all'insegna dell'autonomia e della partecipazione, espressa nella formula "programmazione democratica", con la quale si voleva marcare una netta distanza rispetto alle tendenze burocratiche e centralizzatrici dell'apparato statale [Pci, Comitato regionale Emilia Romagna 1967]. Intorno alla fiducia e all'investimento nella programmazione, le amministrazioni locali costruirono quel nesso tra sviluppo economico e sviluppo della vita democratica che caratterizzò in modo duraturo l'immagine del "modello emiliano".

In questo quadro, si affermò appieno l'importanza dell'urbanistica come strumento di governo. Nel 1963 la giunta comunale di Bologna pubblicò un programma pluriennale per la città e il circondario. Nel documento un capitolo era dedicato alla lotta contro la rendita e vi si spiegava come la presenza di tante aree edificabili avrebbe favorito gli interessi della proprietà immobiliare e non quelli dei lavoratori. Da qui, la scelta della salvaguardia del centro storico, che era preferibile risanare anziché demolire. Giuseppe Campos Venuti, assessore all'urbanistica dal 1960 al 1966, ha ricordato di recente:

Nei fatti Bologna è stato il primo Comune italiano che ha scoperto che si potevano fare investimenti di tipo keynesiano; dal 1960 al 1964 gli investimenti comunali quadruplicarono. Per carità, nessuno nominò mai Keynes, però la cosa era molto semplice: i Comuni avevano una parziale autonomia fiscale e i proventi del prelievo fiscale moltiplicarono i mutui - il deficit spending - e furono investiti in servizi pubblici e case popolari a beneficio di tutti i cittadini, che in grande maggioranza erano dei lavoratori ed elettori del Pci. A noi sembrava tra l'altro che questo impiego del reddito cittadino rispettasse perfettamente la più ortodossa linea del Partito comunista [Campos Venuti 2010, 122-3].

Il partito comunista emiliano attuava, dunque, delle politiche keynesiane. Ma da dove nasceva questo pragmatismo? Probabilmente non dal politicismo di Togliatti; era invece qualche cosa di più profondo, da ricondurre alla storia delle culture politiche della regione, a dimostrazione dell'importanza di riportare il filo del discorso alle radici del riformismo storico emiliano-romagnolo [De Maria 2012]. Fin dai tardi anni Quaranta, nei comuni del territorio guidati dal Pci, si era impostata a livello amministrativo l'immagine complessiva di un "comune del popolo", che riprendeva in maniera impressionante l'identità politica a base territoriale che era stata un pilastro fondamentale del socialismo riformista nei decenni a cavallo del 1900. «Il comune è di tutti i cittadini», affermava in quegli anni il sindaco di Bologna, Giuseppe Dozza, ma «deve però preoccuparsi in particolare modo, del miglioramento generale della città, di sollevare le categorie più diseredate che costituiscono il maggior numero dei cittadini stessi». Un'impostazione 
che si era tradotta subito, grazie all'uso mirato degli strumenti amministrativi, in proposte specifiche: ad esempio, la differenziazione, a vantaggio dei lavoratori, delle tariffe del gas e dei trasporti o l'aumento del minimo imponibile nell'imposta di famiglia.

Il dibattito sul decentramento portò nella prima metà degli anni Sessanta alla nascita dei quartieri, che più tardi sarebbero diventati un pezzo del sistema istituzionale nazionale. Intorno alle strutture assembleari di zona - che ebbero in quegli anni una importanza notevole nell'articolazione delle politiche socio-sanitarie e scolastiche comunali [Lambertini 2012] - avvennero suggestive riflessioni, ricche di sensibilità verso le istanze della democrazia diretta e ancorate all'idea del comune come cellula base della vita democratica del paese. Una delle voci più autorevoli fu quella di Renato Zangheri, che negli anni Settanta intervenne più volte sul tema del decentramento comunale. La sua riflessione storica e politica partiva dall'“impadroniamoci dei Comuni" di Andrea Costa (1883), come «premessa, via via sviluppata, di una linea di azione e di pensiero che è stata ed è patrimonio del movimento operaio italiano, e che in Italia presenta marcati caratteri originali» [Zangheri 1975, 279]. L'obiettivo era quello di mutare la qualità del potere locale ed esisteva chiara consapevolezza tra i comunisti emiliano-romagnoli che facendo questo ci si riconnetteva alle radici del municipalismo popolare ottonovecentesco: al suo sforzo di estendere la presenza delle amministrazioni locali da un puro compito fiscale e di ordine pubblico alla gestione diretta di servizi sociali e al sostegno delle lotte del lavoro. Secondo le parole dell'allora sindaco di Bologna, i consigli di quartiere potevano rappresentare «uno strumento dell'intervento popolare nelle scelte politico-amministrative del comune e nella loro attuazione» e avrebbero permesso di trasformare davvero «la sostanza del potere», dissolvendo l'«aura» della rappresentanza, «per fare di essa una realtà criticabile e controllabile». Si coglieva un riferimento implicito e critico alle frange extraparlamentari, all'estremismo delle quali si contrapponeva il «lavoro minuto» che è alla base del governo di una città:

Il lavoro minuto, apparentemente minore, del consiglio di circoscrizione, dei comitati di gestione delle scuole dell'infanzia, delle biblioteche, degli asili nido, ha portato comunque alla istituzione di un metodo di partecipazione di grande valore democratico. Quando in una città di 100.000 abitanti vi sono nei consigli di quartiere e nei comitati di gestione 7-800 persone che quasi permanentemente partecipano a quelle attività, mentre fino a 5-6 anni fa questo non accadeva e tutto si risolveva nel Consiglio comunale e nella giunta, non possiamo che dare un giudizio positivo di un processo certo non compiuto, difficile e contrastato, ma che è avanzato, che ha aperto spazi ulteriori alla vita democratica, anche in quelle grandi città ove la concentrazione urbana ha creato guasti che avrebbero scoraggiato chiunque; dove si sono ereditate situazioni deteriorate non solo sul piano ammi- 
nistrativo, ma in particolare nel rapporto tra amministratori e cittadini, in tema di partecipazione democratica [Zangheri 1978, 47-8].

Il passaggio dalla programmazione democratica e decentrata del "modello emiliano" alle prime linee di indirizzo della regione Emilia Romagna avvenne lungo il solco tracciato dal paradigma della partecipazione. Si parlava, infatti, di «regione aperta», alludendo a un impegno costante e responsabile a ricercare la «partecipazione degli enti locali e della società civile in tutte le sue articolazioni» e il «confronto fra le forze politiche $[. .$.$] al di là di schieramenti definiti e$ contrapposti $\rangle^{5}$.

Con l'introduzione delle regioni a statuto ordinario doveva chiudersi una stagione, quella caratterizzata dalla lunga battaglia autonomista degli enti locali, e aprirsi una nuova fase di riforma del potere pubblico:

Gli enti locali, nell'articolazione pluralistica dello Stato regionale, vengono ad assumere una collocazione nuova, che deve rappresentare lo sbocco conclusivo della lunga lotta condotta per l'affermazione delle autonomie locali contro l'oppressione del centralismo burocratico.

Nei documenti comunisti di questa primissima fase si incontrava ancora una formula avanzata e di netta rottura, rispetto alla tradizione dello Stato unitario accentrato, come quella di "Stato regionale", che però negli anni successivi scomparirà velocemente a favore di una scelta lessicale più generica e prudente: "Stato delle autonomie". Fin dal 1973, con l'affermarsi della linea del "compromesso storico", emerse con chiarezza la tendenza a subordinare la questione regionale alle dinamiche politiche del paese e al ruolo in esse giocato dal Pci. Anche nel discorso pubblico dei comunisti emiliano-romagnoli lo slancio regionalista sembrava sfumare di fronte a esigenze superiori:

Le regioni, che sono nate nel 1970 come conquista democratica contro gli elementi di crisi in atto nello Stato e nella società e per lo sviluppo, sul piano politico, dell'avanzata del movimento di lotta operaia e popolare, sono oggi chiamate, come condizione della loro stessa vita, a intervenire energicamente e con tutto il loro peso politico e costituzionale a difesa della democrazia e delle istituzioni repubblicane e per lo sviluppo della società. Questo è il compito prioritario che la regione Emilia Romagna si riconosce e si assume, in piena responsabilità, nel presente momento della vita nazionale [Pci, Comitato regionale Emilia Romagna 1973, 1].

Pci, Comitato regionale Emilia Romagna, L'impegno e l'iniziativa unitaria dei comunisti dell'Emilia Romagna per la piena attuazione dei poteri della regione e per avviare la nuova fase di governo regionale, settembre 1971, in FGER, PCI CR-ER, Segretario regionale, b.1 "Sergio Cavina". 
Lo stesso anno, le giunte delle tre "regioni rosse", Emilia Romagna, Toscana e Umbria, conquistate dal Pci nelle elezioni del 1970, furono le prime a elaborare delle piattaforme regionali di sviluppo, sforzandosi di aprire su di esse una consultazioni e confronti pubblici capaci di coinvolgere le varie espressioni politiche e sociali dei loro territori. Ci si trovava all'indomani del primo trasferimento di compiti amministrativi alle regioni, con gli undici decreti delegati del 1972. Era il primo esperimento concreto di una programmazione regionale operativa, $\mathrm{e}$ - più in generale - era il primo tentativo di avviare un processo diverso di programmazione, dopo il sostanziale fallimento della pianificazione nazionale del centro-sinistra.

Il programma quinquennale 1972-77, del quale il governo aveva annunciato la presentazione in parlamento entro la fine del 1972, non soltanto non era stato ancora elaborato, ma non sembrava neppure essere oggetto di quelle attività di studio e di dibattito che sarebbero state necessarie per la sua effettiva messa a punto. Il Comitato interministeriale per la programmazione economica (Cipe) aveva approvato un documento arditamente definito "Piano economico per il 1973", ma nessuno lo aveva ritenuto degno di grande considerazione. Secondo le cronache politiche di quei giorni, era stato presentato all'opinione pubblica con un esame meno che sommario, durato sì e no mezz'ora, e le poche copie dattiloscritte messe a disposizione del parlamento non avevano incuriosito nessuno. In tutto questo, pesavano sicuramente la svolta a destra compiuta dalla Dc tra la fine del 1971 e l'inizio del 1972, e la fine del centro-sinistra.

Nel contesto della crisi della programmazione nazionale, ecco inserirsi il tentativo delle regioni guidate dal Pci. Ciascuno dei tre documenti usciva ormai dall'orizzonte di una pura descrittiva della situazione esistente e dall'ambito di proposte solo infrastrutturali, e scendeva sul terreno di scelte concrete, che puntavano a incidere nell'organizzazione e negli indirizzi dei settori fondamentali dell'economia e dei servizi, e a connettere a queste scelte un nuovo assetto del territorio. Gli interventi previsti per lo sviluppo industriale erano rivolti innanzitutto alla soluzione dei maggiori problemi che stavano dinanzi all'artigianato e alle piccole e medie industrie. Si prevedevano finanziamenti agevolati a favore delle imprese artigiane che si fossero proposte, in forma singola o associata, di elevare il livello produttivo, o quello dei servizi, o dell'ambiente di lavoro, e l'erogazione di contributi in conto interessi per i mutui da contrarre in modo da facilitare alle imprese l'avvio di nuovi rapporti con gli istituti di credito.

Gli interventi previsti per l'agricoltura erano finalizzati a incentivare la produzione di generi suscettibili di conservazione o di trasformazione industriale e al miglioramento delle dotazioni di infrastrutture civili e sociali esistenti nelle campagne. 
La creazione istituzionale più originale fu proposta dalla regione Emilia Romagna con il lanciò di un Ente regionale per la valorizzazione industriale del territorio (Ervit), che avrebbe assunto la forma di una società per azioni a prevalente partecipazione regionale. D'intesa con i comuni e le province, il nuovo ente doveva provvedere alla creazione, nelle zone meno sviluppate della regione, di aree industriali attrezzate da mettere a disposizione delle piccole e medie imprese, cercando di far sì che queste investissero i capitali di cui disponevano, non già in attività immobiliari, ma soprattutto in impianti e attrezzature direttamente produttivi. Inoltre, all'Ervit la regione intendeva assegnare il compito di svolgere una vasta attività di assistenza tecnica, finanziaria, commerciale e amministrativa nei confronti delle piccole e medie industrie.

Il progetto di programmazione regionale presentato da Guido Fanti nel marzo 1973 venne approvato dal consiglio regionale il mese successivo con il voto favorevole non soltanto del Pci e del Psi (i socialisti, benché non fossero ancora entrati nella giunta regionale, erano tornati al governo con il Pci in quasi tutti i grandi comuni della regione), ma anche di Pri, Psdi e Dc. Nel caso dei repubblicani l'adesione alle proposte della giunta comunista non sorprendeva, dal momento che nei comuni romagnoli il Pri si era avvicinato negli ultimi anni alla collaborazione con il Pci, ma sicuramente non era scontato l'appoggio portato da socialdemocratici e democristiani.

Prendendo atto di questo largo consenso l'assemblea regionale decise che i gruppi consiliari avrebbero dato vita a un comitato regionale paritetico di carattere politico-scientifico per approfondire i problemi della programmazione. A livello delle province e dei comprensori, sarebbero poi sorti comitati paritetici analoghi a quello regionale, col compito di stimolare e realizzare la partecipazione di base alla definizione della politica di programmazione (ordine del giorno approvato dal consiglio regionale il 18 aprile 1973).

Perché, è il caso di chiedersi a questo punto, entrò in crisi il processo di rinnovamento istituzionale innescato dalla riforma regionale del 1970? Secondo il dettato costituzionale, la regione avrebbe dovuto presentarsi come un apparato leggero, essenzialmente di programmazione, caratterizzato da un forte coordinamento con gli enti locali. E si è già detto del declino politico e culturale dell'idea di programmazione, consumatosi nel corso degli anni Ottanta. Ma la fase costituente regionale si articolò in vari momenti e almeno fino ai due decreti presidenziali del 1977 che misero in moto il processo di trasferimento dei poteri alle regioni, fu possibile pensare - in una situazione fluida, in cui l'ordinamento regionale doveva essere ancora completato - alla possibilità di realizzare una compiuta riforma in senso autonomista e regionalista del potere pubblico, capace di modi- 
ficare in profondità il quadro dello Stato centralizzato. Tale progetto, alla fine di quel decennio, risultò però incompiuto. Le resistenze furono molteplici: l'inerzia dell'amministrazione ministeriale, la pesantezza della nuova burocrazia regionale, le resistenze delle forze politiche conservatrici, indubbiamente di grossa parte della Dc, ma anche un certo spirito dirigistico prevalso all'interno del Partito comunista [Tarrow 1979b; Bianchi 1987; De Maria 2009].

Le speranze suscitate nel 1970 dalla riforma regionale sembravano ormai relegate in un passato remoto. Nel corso degli anni Settanta l'atmosfera politica e culturale del paese era profondamente cambiata. Il movimento studentesco palesò la tendenza a ritrarsi dall'impegno sociale concreto, per aggrapparsi ossessivamente alla dimensione politico-ideologica, spesso fortemente settaria [Fofi 1994]. C'era l'illusione di una possibile e vicina rivoluzione, ma nei fatti si perse di vista ogni corretta riflessione sul rapporto tra fini e mezzi. Uno smarrimento che ebbe come esito peggiore e senza ritorno quello della violenza politica e della lotta armata. Si perse in larga parte per strada quella spinta alla partecipazione che proveniva dal Sessantotto e dal Concilio vaticano II e che negli anni precedenti aveva animato organismi rappresentativi come i consigli di quartiere o i consigli d'istituto nelle scuole; realtà di base nelle quali era stato particolarmente attivo il mondo femminile.

Il riferimento è, più in generale, a quelle istanze di autogoverno e di partecipazione che, nel 1970, avevano dato slancio al discorso programmatico di Fanti, primo presidente della regione Emilia Romagna, già sindaco di Bologna nel quadriennio precedente:

Attraverso le Province, i comprensori, i Comuni, gli organi decentrati di quartiere, le organizzazioni della società civile [...], attraverso le nuove forme di democrazia di base che sorgono nei luoghi di produzione, occorre riuscire a porre all'ordine del giorno di tutte le forze sociali e politiche l'assetto istituzionale da creare nelle Regioni [...]. Sotto questo profilo, il Consiglio regionale dovrà essere il punto di confluenza di un ampio processo di partecipazione che muova, da un lato, dagli istituti rappresentativi e dall'altro dall'articolazione degli istituti di autogoverno delle masse. Il Consiglio regionale troverà in questo metodo sostanziale, e non già nei caratteri formali, il più vero segno della propria democrazia e della propria funzione sociale [Fanti 1970, 60-2].

Ben diverso sarebbe stato il tono dell'analisi di Fanti agli inizi degli anni Novanta, nelle pagine di un denso articolo con il quale il dirigente comunista emiliano ripercorreva, sul filo dei ricordi, la parabola del regionalismo. Nella sua lettura, era oltremodo significativo che il decreto 616/77 fosse stato realizzato in uno scontro aperto con le resistenze centralistiche che si esprimevano attorno ad alcuni ministeri. La battaglia per l'approvazione del decreto fu così aspra e dura da richiedere 
la seduta del consiglio dei ministri più lunga della storia del paese. All'indomani dell'approvazione del decreto nell'agosto del 1977, ricordava Fanti, Aldo Moro volle sottolineare in un articolo per il "Giorno" il significato profondamente riformatore che poteva avere per lo Stato italiano la piena applicazione di quel provvedimento. Aspettative che furono però interamente disattese:

Nel consegnare il decreto al governo a nome della commissione parlamentare che presiedevo, lo accompagnai con una relazione redatta con il contributo del professor Giannini, allora consulente della commissione stessa, assieme ad altri giuristi come Amato, Reviglio, Bassanini e D’Onofrio. La relazione poggiava su una tesi centrale nella quale si affermava inequivocabilmente come il lavoro di completamento del trasferimento di poteri alle Regioni fosse del tutto inutile, se non veniva accompagnato dalla riforma degli organi centrali dello Stato e dalla riforma degli enti locali. Per la riforma degli organi centrali si rendeva necessaria l'eliminazione di alcuni ministeri, l'accorpamento di altri, lo snellimento delle procedure parlamentari e soprattutto la riforma della Presidenza del Consiglio sulla base di un affidamento al presidente stesso di compiti di indirizzo, di orientamento e di collegamento con le Regioni. Per la riforma degli enti locali si evidenziava la necessità improrogabile di affidare tutta la gestione della parte amministrativa agli enti locali riformati e rinnovati per impedire che anche nelle Regioni si sviluppasse lo stesso elefantiaco burocratismo dello Stato centrale. Queste due riforme essenziali non sono state realizzate ed è ben noto il fallito tentativo compiuto successivamente da Massimo Severo Giannini di applicarle nella sua qualità di ministro. Anzi, tutta la lotta per il rinnovamento e la riforma dello Stato si è di nuovo afflosciata su posizioni addirittura precedenti alla nascita delle Regioni [Fanti 1993, 34-7].

\section{La svolta conservatrice della seconda metà degli anni Set- tanta}

Dopo il 1976 l'esperienza legislativa delle regioni a statuto ordinario conobbe una svolta decisiva. La fase delle "anticipazioni" regionali, cioè della pre-costituzione a livello locale di sedi di intervento settoriale (consorzi socio-sanitari) o di programmazione orizzontale (comitati comprensoriali), si concluse definitivamente. Il cambiamento fu sentito in maniera particolarmente brusca in regioni come l'Emilia Romagna, la Toscana, il Veneto e la Lombardia, dove il progetto di governo regionale si era nutrito di una cultura politica (di matrice socialista o cattolico-sociale) incentrata sui valori dell'autonomia e dello sviluppo locale, caratterizzandosi per una spiccata inventiva istituzionale.

Con il governo Andreotti di solidarietà nazionale si spostò decisamente al "centro", al livello nazionale, l'asse delle iniziative sia per le riforme di settore (basti pensare a quella sanitaria) che per quelle istituzionali generali. Da questo mo- 
mento, e per una moltitudine di ragioni, le trasformazioni vennero prevalentemente giocate a livello romano. Il risultato fu quello di snaturare il ruolo costituzionale delle regioni, non più considerate come enti di governo, per relegarle a divenire enti di pura gestione amministrativa: cioè, strumenti più che attori [Cammelli 1980].

L'esperienza delle "anticipazioni" lasciava residui che in parte potevano essere superati in modo fisiologico e positivo (come la trasformazione dei consorzi socio-sanitari in unità sanitarie locali) ma per altri aspetti rappresentavano una scelta da considerare ormai superata, con particolare riferimento ai comprensori. Mutò anche la "filosofia" che fino a quel momento aveva ispirato il rapporto tra regioni ed enti locali. Mentre per tutta la prima legislatura si era mirato a costituire prima le nuove sedi istituzionali (comunità montane, comprensori, distretti scolastici, consorzi sanitari) per poi trasferire a questi livelli le funzioni corrispondenti (attraverso lo strumento della delega), dalla seconda metà degli anni Settanta il discorso apparve perfettamente rovesciato. Prima il trasferimento di funzioni ai comuni (ad esempio con il decreto 616 e la riforma sanitaria) e poi un limitato riordino del livello municipale, essenzialmente attraverso le associazione di comuni, per la mera gestione dei servizi.

Se è vero che l'art.11 del decreto 616 esprimeva la necessità di legare il conferimento delle deleghe al ruolo di programmazione delle regioni, il suo spirito si scontrò però con le scelte operate a livello centrale che fecero cadere molte delle speranze su cui si era retto l'istituto regionale. Infatti, si andò aggravando, alla fine degli anni Settanta, la settorializzazione delle risorse, anzi la loro micro-settorializzazione, che rendeva le regioni organi decentrati di spesa ministeriale; in alcuni casi, come accadeva per i fondi destinati all'edilizia scolastica, lasciando alle regioni una libertà di manovra addirittura più ridotta rispetto a quella che era stata riconosciuta ai vecchi organi dell'amministrazione centrale, segnatamente i provveditorati regionali alle opere pubbliche [Barbera 1980].

Tra il 1976 e il 1977, questi mutamenti di equilibrio corrisposero con il momento decisivo e più delicato della programmazione regionale: proprio allora, infatti, le giunte regionali erano impegnate nella elaborazione dei primi quadri di riferimento per la programmazione; nella predisposizione dei progetti poliennali di intervento; nell'articolazione dei bilanci per il periodo 1978-81; nell'attuazione della legge 382 e dei relativi decreti. Una complessa azione legislativa e politica che avrebbe richiesto stabilità e fiducia nel rapporto tra società e istituzioni sia a livello locale che nazionale.

Accadeva tutto il contrario. Il Pci usciva molto provato dall'esperienza governativa del 1977, dopo aver sostenuto con l'astensione il governo monocolore 
della Dc. In mancanza di provvedimenti riformatori sul terreno dello sviluppo, del Mezzogiorno, dell'occupazione e dell'organizzazione del lavoro divenne il bersaglio della protesta giovanile, del disagio operaio, della delusione degli strati intermedi e intellettuali.

L'epicentro di questa protesta fu Bologna, nel cuore del "modello emiliano", che proprio sullo scorcio degli anni Settanta cominciò a mostrare vistose incrinature. È stato Fausto Anderlini [2012] a insistere su una periodizzazione del "modello Emilia" che dia centralità alla cesura del Settantasette e all'aspra contestazione giovanile nei riguardi dell'amministrazione bolognese; una contestazione condotta prevalentemente sul piano politico-culturale. Il "modello emiliano" aveva sempre considerato come parte integrante del welfare locale il tema dell'investimento in cultura, ma ora se ne denunciavano una sorta di centralismo e una scarsa apertura rispetto a nuovi contenuti emergenti dalla società civile: insomma, tutto quello che i movimenti extraparlamentari percepivano come risultato del "soffocante" egemonismo del Pci. Una critica che venne in certa misura ripresa e sviluppata, negli anni successivi, anche da settori laici e socialisti [De Maria 2011a]. I comunisti uscirono definitivamente dall'area di governo all'inizio del 1979. Nelle elezioni politiche del giugno di quell'anno il partito perse un milione e mezzo di voti, pari a 4 punti percentuali, calando al 30,4\%. Tanti giovani e variegati gruppi sociali avevano di molto esteso nella tornata elettorale del 1976 il voto al Pci, che ora pagava il conto della delusione prodotta dalla durezza delle condizioni del paese. Per la prima volta cresceva l'astensione: nuovo sintomo di sfiducia e distacco dalla vita politica [Barbagallo 2003].

Nel 1981 la questione morale esplodeva nel cuore dello Stato con il ritrovamento degli elenchi degli iscritti alla loggia P2 nella villa di Licio Gelli. La Dc perdeva per la prima volta la presidenza del consiglio, affidata al repubblicano Spadolini, e al successivo voto politico (giugno 1983) quasi il 5\% dei voti. Ma il Pci non intercettava alcunché di questo riflusso democristiano. Si arrivò, nell'agosto 1983, alla formazione del governo presieduto da Craxi. Il sistema politico degli anni Ottanta sarebbe stato caratterizzato dal protagonismo politico (e insieme dal mancato sfondamento elettorale) del Psi, dall'isolamento e dalla crisi di iniziativa politica del Pci e dal continuo deteriorarsi dei rapporti fra i due partiti della sinistra [Sabbatucci 2003].

Sul piano istituzionale non si realizzò né uno Stato regionale - ché il centralismo continuò come prima ad operare - né una programmazione coordinata con le regioni, né un superamento degli squilibri. Niente, insomma, di assimilabile a una rifondazione vera e propria dei meccanismi istituzionali e socio-economici. Ciò che si verificò fu invece un riassorbimento dei nuovi istituti nei meccanismi 
centralizzati dello Stato con una differenziazione, in tale adattamento, tra le varie regioni in relazione ai rispettivi retroterra storico-sociali (cultura civica dei cittadini, qualità del personale politico-amministrativo, ecc.). In questa parabola l'istituto regionale, quali che fossero le sue originarie istanze, vide sempre più appannarsi il ruolo di entità politica forte sul piano dell'innovazione istituzionale, per assumere crescenti compiti e funzioni di "amministrazione attiva", e in ciò schiacciando, come si è osservato da più parti, il suo profilo su quello tipico di un ente locale. Tutta la trama dei rapporti tra strutture centrali-regioni-enti locali risultò così impoverita rispetto alle iniziali istanze di riarticolazione del sistema democratico italiano. La regione rimaneva a metà del guado, un oggetto-istituto ambiguo, partecipe tanto del centralismo statalista quanto dell'autonomismo localistico [Massari 1987].

Pesò sicuramente il fatto che molta dell'attenzione e delle energie disponibili nel mondo della politica e tra l'opinione pubblica fossero assorbite dai drammatici problemi del terrorismo e della crisi economica; e che, pertanto, rimanesse poco tempo per pensare al riordinamento istituzionale del paese e, in particolare, al sistema delle autonomie.

Maurizio Fioravanti si è chiesto se sia legittimo sostenere che gli anni Settanta, con il loro carico complessivo di problematiche, politiche, sociali e istituzionali, siano stati così rilevanti e incidenti nella storia della nostra democrazia da poter essere indicati come il periodo in cui ha avuto inizio una vera e propria trasformazione del modello costituzionale. Ne ha ricavato una risposta affermativa, secondo la quale si avviò in quel decennio un cambiamento nel modo prevalente d'interpretare la Costituzione e d'intendere il suo ruolo nell'ambito dell'ordinamento giuridico, nella sfera della politica, nella società medesima. Rifacendosi all'opinione di costituzionalisti come Costantino Mortati e Vezio Crisafulli, Fioravanti ha parlato di un crollo verticale dell'attuazione costituzionale per via politico-parlamentare:

È vero che proprio in quegli anni Settanta si deliberano molte leggi di grande rilevanza sociale, che potrebbero essere lette, nel loro complesso, proprio nella linea dell'attuazione costituzionale, e in particolare in quella ispirata dal principio costituzionale di uguaglianza: dallo Statuto dei lavoratori alla tutela delle lavoratrici madri, dal nuovo processo del lavoro alla riforma del diritto di famiglia, dalla istituzione del Servizio sanitario nazionale alla disciplina delle locazioni. Ma è anche vero che questo coincide troppo spesso con l'abbandono di ogni politica di programmazione, con l'avvio della legislazione di carattere congiunturale, con la rincorsa alla legge sugli oggetti più minuti e disparati, sotto la pressione di quegli interessi sezionali che i partiti riescono sempre meno a selezionare, a filtrare, a ricondurre alla dimensione dell'indirizzo politico [Fioravanti 2003, 306]. 
Mutava il rapporto tra politica e Costituzione, nel senso che tendeva a tramontare, a causa della sempre più evidente crisi dei partiti, l'idea di una politica animata da finalità costituzionali. Una crisi di contenuti che si ripercosse sulle regioni.

Giorgio Pastori [1980] poteva intitolare Le regioni senza regionalismo uno dei tanti bilanci fatti in occasione del primo decennio dell'esperienza regionale. Il promesso nuovo modo di fare politica e amministrazione, che pure era stato scritto negli statuti regionali, non si era concretamente manifestato nel modo di essere dei partiti. E ciò mentre, invece, si registrava un progressivo radicamento delle regioni stesse nei valori dell'autonomismo locale e nella coscienza dei cittadini [De Siervo 2003]. Alla crisi delle ideologie politiche stava corrispondendo una forte riscoperta delle identità a base territoriale.

Nel corso degli anni Ottanta, mentre le sezioni dei partiti vedevano calare i loro iscritti (soprattutto tra i giovani) e in alcune regioni settentrionali iniziava a prendere vigore la protesta leghista (sulla scia di un movimento di opinione che rivendicava alle regioni un riconoscimento maggiore, insistendo proprio sulle identità locali), in Emilia Romagna la presidenza di Lanfranco Turci tentò di lanciare una nuova fase costituente (su questo punto si rinvia al par.7). Ma la fortuna politica dell'idea stessa di programmazione era ormai declinante e si udiva invece sempre più spesso echeggiare quell'appello agli "spiriti animali" e al "liberi tutti" dalle regole, latente nella società italiana fin dal "miracolo economico" [Crainz 2009].

\section{Controlli e tributi: le regioni fuori dalla porta}

Dopo quella che fu definita la "seconda fase costituente delle regioni" e che corrispose al periodo di attuazione della legge 382, le risorse finanziarie attribuite alle regioni si andarono sempre più parcellizzando per settori di intervento e vennero rigidamente vincolate dal centro. Le regioni furono sommerse da alcune centinaia di leggine, estremamente dettagliate e contraddittorie, di difficile lettura e interpretazione, e comunque lesive delle competenze regionali.

Le origini del problema risalivano perlomeno alla riforma tributaria del 1971-73 o meglio a quel complesso normativo comunemente identificato come "riforma tributaria". I provvedimenti dei primi anni Settanta avevano, infatti, raccolto in pieno le spinte accentratrici seguite - come di riflesso - all'istituzione delle regioni, distruggendo completamente l'autonomia fiscale degli enti locali:

Non è dato sapere del tutto se per calcolo preciso o per caso dovuto a velleità tecnocratiche, mentre si completava l'ordinamento costituzionale della Repubblica italiana, mediante l'istituzione delle Regioni a statuto ordinario e il concreto avvio del decentramento amministrativo e politico dello Stato, gli enti locali territoriali 
venivano privati di ogni potestà in materia tributaria e ridotti nella totale soggezione finanziaria al potere centrale, cosa che non era accaduta nemmeno sotto l'imperio delle politiche più spiccatamente accentratici di epoca liberale e di epoca fascista [Martinelli 1983, 29].

Scompariva quel tanto di autonomia impositiva degli enti locali, e in particolare dei comuni, che aveva costituito una costante nella storia italiana. Contemporaneamente si realizzava il più ampio decentramento politico-amministrativo che la storia del nostro paese avesse mai conosciuto. E poiché le scelte politiche e quelle amministrative comportano sempre l'effettuazione di spese, il decentrare significa di norma moltiplicare i centri di spesa. Viceversa, cancellare l'autonomia tributaria aveva l'effetto di trasformare la politica dell'entrata in una elencazione di richieste allo Stato il cui costo complessivo rappresenta il fabbisogno finanziario dell'ente. Era palese la contraddizione esistente nell'eliminare ogni capacità impositiva agli enti locali proprio nel momento in cui si ampliava la loro sfera di azione e si riducevano i controlli, soprattutto i controlli prefettizi. Ma, a ben vedere, esisteva una logica profonda in quanto stava accadendo. Il controllo sugli enti locali, infatti, era stato per oltre un secolo nelle mani dei prefetti e aveva rappresentato lo strumento di una lunga e paziente mediazione fra livello nazionale e livelli locali del sistema politico. A partire dal 1970, staccata la funzione di controllo dal prefetto, e venuto meno in buona parte il ruolo da questi tradizionalmente svolto, chi avrebbe provveduto a coordinare il governo locale con il restante assetto istituzionale? Si stavano strutturando, è vero, i "comitati regionali di controllo", cui sarebbe spettato il controllo di legittimità sugli enti locali, ma essi rispondevano all'ente regione e non allo Stato.

In sostanza, come conseguenza dei provvedimenti del 1971-73, gli enti locali dopo aver elaborato e deliberato i propri programmi sociali giravano, letteralmente, al governo il conto di tali operazioni, scaricando verso il centro l'insoddisfazione o, talvolta, la rabbia popolare per i tagli che si sarebbero dovuti apportare ai programmi iniziali a causa dell'impossibilità di finanziare alcune delle spese programmate. Si instaurò, insomma, una tendenza alla separazione tra centri decisionali della spesa e provvista delle entrate, che finì per favorire un atteggiamento irresponsabile dei ceti dirigenti locali rispetto ai bilanci, con quel che ne seguì in termini di allargamento della spesa [Istat 2011].

A una situazione siffatta si aggiunsero i provvedimenti sulla finanza locale del 1980-81. I contenuti di questa "miniriforma" denotarono una perfetta saldatura fra la diffidenza antiregionalistica dei rappresentanti degli enti locali e gli umori antiregionalistici degli apparati centrali [Emiliani 1981; Onida 1981]. In un sistema delle autonomie che assegnava all'ente regione il compito della program- 
mazione generale del territorio e che quindi implicava, per la gran parte della spesa degli enti locali - quella almeno che era spesa di intervento e riequilibrio del territorio -, non una semplice presa di visione ma un attivo intervento di programmazione regionale, era paradossale constatare che se c'era una cosa che in vari anni di contrattazioni e di scontri sui problemi della riforma delle autonomie e della finanza locale aveva accomunato governo e associazioni degli enti locali era proprio la ferma determinazione di lasciare, comunque, le regioni fuori dalla porta. Le regioni non avevano voce in capitolo neppure sull'impiego delle risorse disponibili presso la Cassa depositi e prestiti: vigeva una assoluta separatezza fra finanza regionale e finanza locale, una condizione non riscontrabile in altri ordinamenti decentrati e in palese violazione dell'art.119 della Costituzione.

\section{II livello intermedio di governo: comprensorio o provincia?}

La pianificazione a scala subregionale costitù negli anni Sessanta e Settanta un tema di ampio confronto tra le forze politiche e culturali impegnate sui problemi del territorio. Le motivazioni di quel dibattito nascevano dall'inadeguatezza della dimensione regionale di fronte all'esigenza di un organico processo di pianificazione territoriale e dalla contemporanea necessità di aggregazione rispetto a interventi e servizi che su scala comunale risultavano scarsamente significativi o convenienti. Le due questioni della disaggregazione subregionale e dell'aggregazione sovracomunale sembrarono trovare risposta nell'area strategica del comprensorio, più circoscritta e omogenea rispetto al disegno delle province. $\mathrm{Ne}$ seguì una polemica sulla legittimità dei nuovi ambiti territoriali e dei relativi strumenti di pianificazione che superavano le divisioni amministrative tradizionalmente note e costituzionalmente riconosciute [De Marchi 1981].

Fu una breve stagione quella dei comprensori, che almeno inizialmente si pose in sintonia con orientamenti nazionali che prevedevano (dopo l'introduzione delle regioni) il superamento dell'ente provincia, ma che ben presto si esaurì anche di fronte alle difficoltà di organizzare nuovi apparati sufficientemente qualificati e in grado di far fronte a compiti di programmazione e coordinamento.

Incertezze e affanni che, all'inizio degli anni Ottanta, si tradussero in un profondo ripensamento sul significato dell'ente intermedio e sul destino dei comprensori, che scomparvero velocemente. La scelta di rivalutare le province derivò dalla opportunità di valorizzare una istituzione già esistente e garantita dalla Costituzione e di utilizzare un patrimonio di esperienze e di strutture che, pur richiedendo un grande impegno di riqualificazione, comunque esisteva. Le province, inoltre, erano sostanzialmente gradite ai partiti perché garantivano un raccordo immediato 
con momenti dell'organizzazione politica, sociale ed economica tradizionalmente coincidenti con questa area.

Il rischio concreto fu quello di dismettere e abbandonare il positivo lavoro di indagine sul territorio che aveva accompagnato la riflessione sui comprensori. L'esperienza comprensoriale era iniziata ufficialmente nel 1974 in Emilia Romagna con la prima legge regionale in materia; provvedimento che fu ripreso negli anni successivi da quasi tutte le altre regioni (l'eccezione più importante è quella della Puglia, che non istituì i comprensori).

In Emilia Romagna il nuovo istituto raccoglieva l'eredità dei piani intercomunali di coordinamento (Pic) che si erano sviluppati spontaneamente negli anni Sessanta. Proprio in virtù di quella matrice originaria, ai comprensori furono attribuiti vasti poteri e incombenze in materia urbanistica. Una caratteristica che si riscontrò anche altrove, e spesso con ricadute positive per il territorio. In Toscana, ad esempio, nel giro di pochi anni, grazie allo stimolo proveniente dalla regione e dai comprensori, vennero redatti e approvati tutti i 287 piani regolatori comunali, mentre nei trent'anni precedenti ne erano stati introdotti appena 26 [Bortolotti 1986; Istituto regionale programmazione Toscana 1979].

Si trattava di segnali che testimoniavano del fatto che gli aspetti positivi dell'esperienza regionale, pur non sempre visibili a prima vista, sicuramente esistevano: l'irrobustimento del governo locale, una articolazione del potere pubblico certamente più diffusa nella società di quanto non lo fosse nel periodo del centralismo esasperato, la realizzazione di esperienze pilota che avrebbero potuto essere utilmente generalizzate [Bianchi 1986]. Con il 1970 si fecero strada nuove tematiche: ad esempio, l'ambiente e i beni culturali, nel quadro di una riappropriazione del territorio e della cultura da parte della comunità locali.

Le regioni si trovarono di fronte una quantità di problemi nei vari settori fino a quel momento governati, in modo generalmente poco efficiente, dai ministeri o dai loro organi di decentramento burocratico. La necessità di recuperare i ritardi spinse a un eccezionale sforzo di approfondimento nella conoscenza delle situazioni di fatto e del funzionamento di particolari settori produttivi o di particolari aree. $\mathrm{E}$ in questo lavoro sul campo i comprensori giocarono un ruolo significativo. Tuttavia, tra la fine degli anni Settanta e i primi anni Ottanta, con le province ancora saldamente in funzione, i progetti di riforma che impegnarono governo e parlamento concordarono sulla necessità di una semplificazione e riduzione dei livelli amministrativi e di un alleggerimento delle procedure di esercizio delle funzioni locali. Si optò, così, per un solo livello di governo intermedio tra regione e comuni: la provincia, di cui peraltro si auspicava una profonda riforma [Urbani 1981; Vandelli 1982]. 
In modo simile al comune, anche la provincia avrebbe dovuto delinearsi come "ente pubblico a fini generali", e non più settoriali, riassorbendo di fatto il ruolo che era stato attribuito ai comprensori. Il suo compito sarebbe stato quello di articolare sul territorio la programmazione regionale: garantire, cioè, il coordinamento dei programmi comunali e il collegamento fra regioni ed enti locali in relazione a programmi di sviluppo e a programmi di settore.

La mancanza di un disegno istituzionale preciso era evidenziata dal fatto che il decreto 616 del 1977 aveva ignorato pressoché completamente la provincia, pur nel contesto di massicci trasferimenti di funzioni ai poteri locali, chiamandola in causa tutt'al più per partecipare a qualche procedimento facente capo alla regione. Ci si trovava ancora nella fase della sperimentazione comprensoriale e del dibattito che la accompagnò; per questa ragione, comprensori, comunità montane, circondari e comuni costituivano i soli punti di riferimento presi in considerazione per articolare e decentrare funzioni a livello locale.

Nel giro di due o tre anni, come detto, il dibattitto subì una svolta notevole e le stesse leggi regionali cominciarono ad assumere la provincia come importante destinatario di deleghe, per l'esercizio di funzioni di livello sovracomunale: particolarmente in materia di inquinamento idrico, caccia, pesca, trasporti, formazione professionale e impiego delle terre incolte.

Il convegno nazionale dell'Anci tenutosi a Viareggio nell'autunno 1980 accolse con favore i nuovi indirizzi istituzionali: i sindaci concordarono sul fatto che la provincia, fino ad allora titolare di competenze settoriali e frammentarie, andasse «trasformata in ente intermedio di programmazione» ${ }^{6}$. Del resto, l'abolizione dei comprensori non poteva che essere accolta con favore dall' Anci in quanto indeboliva di fatto l'interventismo regionale e implicava un potenziamento delle capacità operative dei comuni, incentivando la possibilità di esercitare funzioni in forme collaborative (protocolli di intesa, gestioni e associazioni) con altre amministrazioni comunali, sempre in attesa che si ridefinissero i compiti della provincia.

Il rafforzamento dell'ente provincia non mancava di una logica e di una coerenza rispetto alla sua vocazione storica. Se è vero, infatti, che i comuni capoluogo e le grandi città non avevano mai mostrato molto interesse al tema del coordinamento con l'ente intermedio, si può invece osservare come, nel secondo dopoguerra, le province italiane avessero cercato e trovato una propria fisionomia, un proprio preciso ruolo, nel privilegiare il rapporto con i comuni minori presenti sul loro territorio. 
Ma un ragionamento sulle funzioni di collegamento dell'ente provincia portava molto più indietro nel tempo, fino al progetto di riforma amministrativa presentato da Marco Minghetti in parlamento nel marzo 1861: una proposta di articolazione regionale, dove la provincia ricopriva il ruolo di perno del decentramento amministrativo. In realtà la storia istituzionale del nostro paese era andata in un'altra direzione, quella tracciata dall'ordinamento delle amministrazioni locali disegnato nel 1865, che avrebbe dato alle province poche competenze settoriali; le quali si sarebbero assottigliate ulteriormente in seguito, e soprattutto durante il fascismo, quando le province entrarono in un binario morto. La creazione, nel 1925, dell'Opera nazionale maternità e infanzia le privò della gestione dell'assistenza agli illegittimi; nello stesso modo, l'estendersi delle pratiche assistenziali attraverso altri enti, quali il Partito nazionale fascista e l'Opera nazionale balilla, ebbe l'effetto di marginalizzare sempre più il loro ruolo; si arrivò così al 1934, quando fu emanato quel testo unico della legge comunale e provinciale che, con poche modifiche, sarebbe rimasto in vigore fino al 1990 [Aimo 2007 e 2009; Polsi 2004; Rotelli 1991].

La questione dell'ente intermedio di programmazione apertasi tra anni Settanta e Ottanta rimase, infatti, senza soluzione: semplicemente si abolirono con un colpo di spugna i comprensori (frutto della creatività istituzionale delle regioni), senza che arrivasse tempestivamente una riforma complessiva del governo locale.

In Emilia Romagna, la regione che li aveva fondati, i comitati comprensoriali vennero soppressi con legge regionale nel 1982. Ma la regione nella quale si registrarono più resistenze ad abbandonare la strada dei comprensori fu il Veneto. Anche qui l'istituzione del nuovo ente intermedio era stata ispirata, intorno alla metà degli anni Settanta, da un disegno riformatore molto sentito, che portò all'inizio del decennio successivo alcuni esponenti di spicco del governo regionale a opporsi alla cancellazione di quella esperienza.

Tra loro il vice presidente della giunta regionale, il democristiano Marino Cortese [1981], secondo il quale la scelta comprensoriale conteneva in sé una potenzialità di rinnovamento delle istituzioni a cui non si sarebbe dovuto rinunciare. In Veneto, il tema del comprensorio era maturato come "una via obbligata", in conformità con la scelta essenziale prefigurata nello statuto regionale, che voleva una "regione della programmazione e delle autonomie". Conviene ricordare quale fosse la novità istituzionale sottesa a questa scelta. Il comprensorio era considerato come la chiave di volta per l'uscita dalle strettoie e insufficienze della vita comunale: un punto di aggregazione sovracomunale intorno al quale appariva possibile e conveniente programmare lo sviluppo, definire l'assetto territoriale, attuare la politica delle deleghe, promuovere e organizzare una razionale gestione dei servizi. 
Il problema veniva posto all'incirca in questo modo: come conciliare la domanda di spazi e di strumenti istituzionali nuovi, che richiamano alla sovracomunalità, con i valori ereditati dalla storia e dalla radicata esperienza sociale e civile del comune? Si partiva dunque dal peso rilevante che aveva la tradizione del municipalismo cattolico, la quale riconosceva nel comune il punto di maggiore consistenza della vita pubblica, lo strumento di promozione dei più interessanti fatti partecipativi, insomma il fattore insostituibile per la salvaguardia del processo democratico nel paese. Ma era altresì culturalmente percepita l'esigenza di frapporre tra il comune e lo Stato una serie di corpi territoriali intermedi.

Storicamente, l'istanza comprensoriale era maturata in Veneto negli ultimi cinquant'anni, attraverso tutta una gamma di esperienze che facevano riferimento ai consorzi fra i comuni, e fra comuni e province, nell' «antica» ricerca di livelli sovracomunali per superare le insufficienze operative e finanziarie nell'approntamento dei servizi locali [Cortese 1981, 53]. A ben vedere, infatti, la questione di una pianificazione sovracomunale poteva ben dirsi antica, dal momento che si era posta ovunque, in Italia e in Europa, fin dai decenni a cavallo del 1900, in corrispondenza dei fenomeni di urbanizzazione e crescita delle città [Bottini 2003]. A partire dagli anni Trenta, si era andato progressivamente sviluppando il ricorso allo strumento del consorzio per la costituzione di aggregazioni monofunzionali tra comuni e province, che potevano avere scopi organizzativi (per il segretario comunale, per il medico condotto, ecc.), di servizio (per le strade, gli acquedotti, la vigilanza urbana, ecc.), fino alla scoperta dei consorzi di sviluppo (per le zone industriali, per lo sviluppo economico e sociale, ecc.), veri e propri consorzi polivalenti. Tra anni Cinquanta e Sessanta si erano profilate nuove esigenze, come il problema dello sviluppo complessivo del territorio e la programmazione, mentre si approfondivano gli studi sul territorio intrapresi da molte province, per impostare correttamente i problemi dello sviluppo e del riequilibrio. In questi studi non erano mai mancati i primi profili di un assetto per comprensori.

La legge 80/1975 della regione Veneto sui comprensori si era posta al centro di questo dibattito. Le sue scelte, collegate a quelle della legge 64/75 sui consorzi socio-sanitari e della legge $21 / 75$ sui piani zonali per l'agricoltura, erano chiare e coraggiose: il comprensorio come livello di governo, con compiti di programmazione economica, territoriale e urbanistica; strumento di promozione e coordinamento delle politiche di settore e di gestioni dei servizi; insomma, il punto di sintesi delle autonomie locali entro gli orientamenti e le direttive della programmazione regionale.

L'ambito comprensoriale era apparso sempre più chiaramente come lo spazio naturale della politica subregionale, contro ogni tentazione di riprodurre, entro 
l'ambito della regione, le tendenze al centralismo così contestate nei riguardi dello Stato. Il decentramento della programmazione avrebbe permesso di sviluppare la grossa orditura delle indicazioni programmatiche regionali in soluzioni più dettagliate e operative, ma nello stesso tempo non frantumate nella miriade degli ambiti comunali.

Il fatto che i 49 comprensori veneti non fossero decollati (come del resto i comprensori delle altre regioni) era da ricondurre alle difficoltà oggettive insite in quel disegno riformatore - la strutturazione sul territorio di nuovi enti intermedi - ma anche ai condizionamenti derivanti dai ritardi della legislazione nazionale a causa dei quali i comitati comprensoriali non erano stati in grado di operare con sufficiente disponibilità di mezzi e di poteri. Tutto questo, secondo la presidenza della giunta veneta, non doveva essere argomento per un giudizio di condanna e di abbandono, ma doveva indurre al limite a un supplemento di riflessione. Rimaneva il fatto che non si sarebbe arrivati «a varare una vera e significativa riforma degli enti locali territoriali, privilegiando ora la provincia dopo averla trascurata a favore della prospettiva costituita dai comprensori» [Cortese 1981, 55].

Nel programma regionale di sviluppo approvato con legge 11/1979, la giunta del Veneto aveva in qualche modo rilanciato cercando nel contempo una mediazione con gli orientamenti nazionali favorevoli alla provincia. Si arrivò, cioè, a ipotizzare una articolazione di livelli istituzionali in base alla quale si riteneva "troppo semplificatorio" prevedere un solo organo intermedio tra comune e regione.

I comprensori avrebbero continuato a far parte dell'architettura istituzionale subregionale, ma con competenze più limitate (di servizio e non di programmazione), e sarebbero stati affiancati da un altro livello intermedio (di programmazione): quello rappresentato dalle "aree vaste". Nulla impediva che queste ultime potessero chiamarsi "province", ma dovevano essere più numerose e diverse (nelle funzioni e nel disegno) rispetto alle province esistenti, le quali erano destinate all'abolizione.

Il Veneto ribadiva l'esigenza di individuare momenti dimensionali più omogenei, a fronte di una situazione italiana che presentava 8.000 comuni e 94 province. Tale realtà si rispecchiava pienamente nel territorio veneto: nei suoi 582 comuni e nelle sue sette province, le quali avevano delimitazioni interessanti e difendibili solo da un punto di vista storico. Al contrario, dal punto di vista economico e sociale, e per quanto riguardava i poli di urbanizzazione e comunicazione, esse risultavano decisamente superate.

Entrando nel dettaglio del programma regionale veneto, al livello costituito dall'area comprensoriale sarebbe toccata la gestione dei servizi: scuola, assistenza e sanità. L'area vasta, invece, avrebbe aggregato comprensori contigui (all'in- 
circa nel numero di quattro), svolgendo funzioni di programmazione territoriale ed economica, con particolare riguardo alla problematica connessa con la riconversione e ristrutturazione industriale.

Grazie a questa architettura del governo locale - per la cui applicazione si rimaneva naturalmente in attesa che il problema del livello intermedio di governo fosse definito in sede nazionale - secondo la giunta veneta non si sarebbe vanificata l'esperienza politica e amministrativa dei comprensori; essi anzi avrebbero costituito il punto di partenza per comporre, attraverso ben calibrate aggregazioni, l'area vasta di coordinamento intermedio. «Che questa si chiami ancora provincia, è solo questione nominale. Questione sostanziale è invece che l'area intermedia non sia l'attuale provincia» [Cortese 1981, 56].

In definitiva, dal Veneto arrivava un messaggio chiaro: innovazione istituzionale e programmazione passavano attraverso l'esperienza comprensoriale. Sopprimerla avrebbe significato, in sostanza, affossare le istanze di programmazione regionale. Il ritorno di fiamma sul ruolo delle amministrazioni provinciali non veniva interpretato come lo sforzo di ridare vita a un ente a competenze limitate, che troppo poco aveva partecipato al processo sociale. Vi si rintracciava piuttosto la rivincita della provincia come maglia geometrica uniforme del decentramento statale (prefettura, questura, intendenza di finanza, provveditorato agli studi, ecc.) sulla quale si era venuta modellando fin dalla fine dell'Ottocento l'organizzazione della vita politica e sociale - dai partiti ai sindacati, alle associazioni di categoria - dello Stato unitario accentrato.

\section{Alla ricerca di una «programmazione possibile»}

All'inizio della terza legislatura, la giunta regionale dell'Emilia Romagna si impegnò a lanciare una nuova fase costituente, dopo le due che avevano caratterizzato gli anni Settanta. Nel dibattito interno si puntarono i riflettori principalmente sul versante fiscale e su quello delle riforme istituzionali.

Per levare finalmente gli ormeggi alla riforma regionale era necessario, da una parte, dare voce alle regioni a Roma, «trasformando il Senato in Camera delle Regioni, sul modello tedesco», dall'altra riconoscere apertamente alle regioni il potere di operare scelte politiche: questo presupponeva l'attribuzione, sia alle regioni che ai comuni, di una vera autonomia finanziaria e impositiva, coordinata 
fra i due livelli?.

Da tempo, all'interno del Pci, a livello locale come a livello nazionale, esisteva piena consapevolezza sul fatto che l'esperienza della programmazione regionale dovesse sollecitare sviluppi costituzionali innovativi e, in particolare, una riflessione sulle forme e sui contenuti del bicameralismo. Temi cruciali come il bilancio dello Stato dovevano essere oggetto di una discussione cui potessero partecipare le regioni ed era urgente, secondo le parole di Pietro Ingrao [1973], «un rapporto tra i diversi livelli di assemblee [regionali e nazionali], che è tuttora inesistente oppure è del tutto casuale».

Ogni percorso riformatore si bloccò nella seconda metà degli anni Settanta, lasciando in eredità una situazione che appariva fallimentare non solo a esperti e osservatori, ma anche agli occhi degli stessi protagonisti del governo regionale. Tra loro l'esponente modenese del Pci Lanfranco Turci, presidente della giunta emiliano-romagnola, che stigmatizzò la riduzione di fatto dell'ente regione ad agenzia di spesa dello Stato. Le regioni erano organi di programmazione mancati e assomigliavano piuttosto a enti di gestione parastatale. I dati del 1980 parlavano chiaro: su 19.443 miliardi di trasferimenti dallo Stato solo 1.800 potevano ritenersi affidati alla piena autonomia di spesa delle regioni, a cui era affidata in sostanza una prevalente funzione allocativa di risorse predeterminate.

Era evidente l'enorme difficoltà di esprimere politiche realmente incidenti sullo sviluppo del sistema regionale, quando quasi 1'80\% delle risorse a disposizione delle regioni era assorbito dalla spesa sanitaria e le destinazioni di risorse vincolate da leggi statali arrivavano all'incirca al 90\%, sì che agli interventi economici si poterono destinare tra anni Settanta e Ottanta volumi di spesa irrisori rispetto all'entità delle grandezze macroeconomiche regionali con cui si dovevano confrontare. Attraverso le carte della presidenza di giunta conviene approfondire proprio il frangente dei primi anni Ottanta, quando il dibattito all'interno dei vertici regionali sembra toccare aspetti decisivi. La fase di rodaggio dell'ente regione poteva dirsi conclusa e il contesto generale, in Emilia Romagna, era meno negativo che altrove. A livello economico, infatti, la regione viveva un momento relativamente favorevole: erano gli anni nei quali gli organi di informazione parlavano frequentemente di fallimento del modello economico torinese (produzione di massa standardizzata) e di vittoria del "modello emiliano", fatto di produzione specializzata, piccole-medie aziende e collaborazione con il sindacato [Vaudagna 1983]. 
Dagli esponenti di spicco della sinistra emiliana venivano sollecitazioni politiche incisive. Se dunque all'interno del Pci si indicava solitamente nella crisi della politica di solidarietà nazionale il motivo fondamentale che aveva portato a una interruzione lungo il percorso di completa attuazione dello «Stato delle autonomie delineato nella Costituzione» ${ }^{8}$, questa diagnosi era notevolmente arricchita e problematizzata da Renato Zangheri che, fin dal 1978, aveva messo in rilievo la mancanza della necessaria determinazione da parte dei comunisti sui problemi relativi all'area delle autonomie, intravedendo «un certo attendismo» nel suo partito?.

Proprio in quel periodo, i più attenti commentatori politici cominciarono a rendersi conto di una netta sotto-rappresentazione del "partito emiliano" negli organismi dirigenti centrali. Nel tentativo di rispondere alla sconfitta del regionalismo, la giunta regionale guidata da Turci elaborò nel 1981 una relazione sui problemi del riordino istituzionale, nella quale tornava con decisione la centralità del rapporto tra autonomia e programmazione, vero e proprio asse genetico del progetto di governo regionale. Si riteneva che la riproposizione del ruolo delle regioni dovesse avvenire con la forza adeguata alla gravità della crisi istituzionale:

Non ci sentiamo affetti da un'ottica rivendicazionista nel riproporre questi temi [...]. Coniugare autonomia e programmazione è ancora il punto fondamentale che dà un senso reale all'istituzione delle regioni, alla costituzione del sistema delle autonomie. Questa convinzione è sempre stata profondamente radicata nell'azione della regione Emilia Romagna ${ }^{10}$.

A maggior ragione, dopo il trasferimento di poteri a regioni e a enti locali attuato con il decreto 616 e con la riforma sanitaria, occorreva dare attuazione senza indugio alle condizioni che facevano del metodo della programmazione «uno strumento reale di governo»e, in primo luogo, «il modo veramente nuovo e originale di essere della regione».

La giunta emiliano-romagnola ribadiva il principio costituzionale secondo il quale la regione doveva esercitare compiti di legislazione, di programmazione e di alta amministrazione. Da ciò derivava la limitazione delle funzioni di amministrazio-

Regione Emilia Romagna, Consiglio regionale, Proposta programmatica del gruppo Pci per la formazione della Giunta regionale, bozza ds., 22 luglio 1980, in ASRER, Presidenza della Giunta, Segreteria del Presidente, Programmazione e affari istituzionali, b.72.

\footnotetext{
Pci, Comitato regionale Emilia Romagna, riunione del 22 marzo 1978, verbale ms., in FGER, PCI CR-ER, Segreteria, b.2. f.1978.
}

Regione Emilia Romagna, Giunta regionale, Relazione sui problemi del riordino istituzionale, febbraio 1981, in ASRER, Comitato comprensoriale di Bologna, b.10. 
ne attiva alle sole scelte di scala regionale, o di grande scala subregionale, mentre tutto il resto doveva essere delegato agli enti locali. L'esercizio diretto di funzioni amministrative da parte della regione - sia attraverso propri apparati periferici, sia attraverso enti strumentali - doveva diventare l'eccezione e non la regola.

E tuttavia, a fronte dell'auto-percezione e della retorica politica della giunta regionale, va rilevato che i dati sulla spesa reale continuarono a restare sostenuti. Si può affermare che fosse soprattutto il passaggio della gestione della sanità alle regioni a vanificare qualsiasi illusione di programmazione "leggera", rendendo non solo burocratico, ma forzatamente gerarchico, l'intero sistema.

In una riunione di giunta del novembre 1981, dedicata alla definizione del secondo programma regionale di sviluppo (1982-85), l'assessore ai servizi sociali Pier Luigi Bersani invitava ancora una volta a interrogarsi sui motivi per i quali «la regione, al momento in cui ha ricevuto le competenze, non ha trovato la strada per darsi una propria identità, una caratterizzazione»" .

Nei primi mesi del 1982 venne completato il "quadro di riferimento" relativo al piano poliennale 1982-85. L'incipit del documento aveva un tono evocativo, che evidenziava la consapevolezza di trovarsi di fronte a un passaggio decisivo:

Un senso di precarietà, la mancanza di progettualità, orizzonti limitati ad una affannosa ricerca di tamponamento provvisorio delle questioni: tutto ciò caratterizza gran parte della vita italiana. In questa situazione la Regione Emilia Romagna si appresta ad elaborare il secondo piano regionale di sviluppo per i prossimi quattro anni: dal 1982 al 1985. Programmare per il medio periodo è sempre impresa difficoltosa, ancora più nel nostro paese, travagliato da una grave crisi economicasociale-morale e politica [Regione Emilia Romagna 1982].

Il richiamo alla «crisi», non solo economica, ma anche sociale e morale mostrava di non eludere il problema posto al sistema dei partiti dal declino della partecipazione politica. Un fenomeno che si stava registrando in quegli anni e che era da ricondurre alla caduta di tensione, al riflusso, che si percepiva nei processi di trasformazione della società e dello Stato: crisi delle istituzioni e crisi del sociale procedevano e si condizionavano vicendevolmente ${ }^{12}$.

Nuove forme di attivismo e intervento sociale nascevano e si sviluppavano, scegliendo però di dirigersi in altre direzioni, sostanzialmente fuori dalla società politica e dal mondo dei partiti: volontariato, cooperazione sociale, la galassia di

Regione Emilia Romagna, Giunta regionale, riunione del 26 novembre 1981, verbale ds., in ASRER, Presidenza della Giunta, Segreteria del Presidente, Programmazione e affari istituzionali, b.69.

L. Turci, Relazione al convegno Riformare il potere locale per realizzare lo Stato delle autonomie, Milano, 8-9 febbraio 1980, bozza ds., cit. 
associazioni raccolte in quel contenitore denominato "terzo settore", che iniziava a interpretarsi come un vero e proprio movimento. Mutamenti e nuove opportunità che, ben presto, avrebbero spinto a ripensare, nel quadro della crisi fiscale dello Stato, le tradizionali categorie del "pubblico" e del "privato" nella tutela dei diritti sociali [De Maria 2011b].

In un contesto in rapida trasformazione, la strada indicata dal quadro di riferimento del 1982 era quella di una "programmazione possibile»; un approccio che non pretendesse di essere onnicomprensivo, ridisegnando l'assetto sociale ed economico, o tantomeno sostituendosi al mercato, e che tuttavia non si riducesse a un atteggiamento puramente amministrativo:
Nel momento in cui proponiamo la programmazione quale strumento per il go- verno della crisi, abbiamo ben presente il fallimento dei passati tentativi di pro- grammazione nel nostro paese e tutti quegli elementi che caratterizzano negati- vamente la realtà politica presente: il non governo, il deficit pubblico, il livello di inefficienza e di autoriproduzione della pubblica amministrazione. [...] Lo stesso concetto di programmazione va precisato alla luce delle esperienze passate e delle trasformazioni che hanno reso più complessa la nostra società. [...]. Ormai l'espe- rienza ci ha resi immuni da quell'ottimismo preconcetto che era largamente pre- sente tra le forze politiche che sostenevano la programmazione. La ridefinizione di una programmazione «possibile» deve prendere le mosse dalla coscienza dei profondi processi di trasformazione in atto nella società e della loro complessità; una programmazione onnicomprensiva finisce col negare la complessità e la plu- ralità dei bisogni, mentre un atteggiamento puramente amministrativo non riesce a compiere l'indispensabile azione di semplificazione e selezione, finendo con il sovrapporsi al sociale senza governarlo [Regione Emilia Romagna 1982, 5].

Molta acqua era passata sotto i ponti rispetto alla piattaforma regionale di sviluppo del 1973, un piano elaborato prima della crisi del petrolio e dell'amaro risveglio dai sogni di uno sviluppo facile, continuo, illimitato. E tuttavia, pur partendo dalla necessità di fare i conti con le risorse disponibili, il quadro di riferimento del 1982 puntava su tre linee di intervento molto chiare e ancora attuali, imperniate sulla qualità della spesa pubblica e dello sviluppo economico: 1) sostenibilità dell'apparato produttivo, 2) risanamento ambientale, 3) riqualificazione urbana. Sull'onda del rinnovato slancio sviluppatosi intorno all'idea di una terza fase costituente, e alla possibilità che sembrava offrirsi di nuove vie per la programmazione regionale, nacque a Bologna nel 1980 la rivista "Regione e governo locale", che aveva tra i suoi principali animatori Augusto Barbera e Luciano Vandelli. Non era un semplice strumento tecnico (come poteva far pensare il sottotitolo: «bimestrale di documentazione giuridica della Regione Emilia Romagna»), ma fin dal primo numero ebbe l'ambizione di porsi quale «momento di dibattito, di mobilitazione e di partecipata crescita culturale». 
Per pensare a un rilancio del regionalismo era necessario che la regione degli anni Ottanta si rivelasse diversa da quella che si era presentata al giudizio degli elettori dopo un decennio di attività. Conveniva ripartire dalle parole: "autonomia", "autogoverno", "programmazione", spesso usate a sproposito, e dai loro significati [Barbera 1980].

Se il termine "autonomia", ad esempio, aveva ancora un senso - e, cioè, esclusione di ogni dipendenza di tipo burocratico, gerarchico e finanziario - non era possibile applicarla a sistemi di governo nei quali solo la decima parte delle somme disponibili era a utilizzazione discrezionale.

Nello stesso modo risultava problematico parlare di "autogoverno", termine con il quale si presupponeva una completezza nei poteri pubblici corrispondenti a un determinato livello istituzionale. Tutto il contrario accadeva per le regioni, che non potevano contare neppure su un coordinamento efficace con realtà come Anas, Enel, Ferrovie, o altre partecipazioni statali. Le regioni, cioè, non erano chiamate a esprimersi su rilevanti decisioni di spesa del settore pubblico "allargato", che pure incidevano sullo sviluppo regionale.

Si trattava di situazioni senza precedenti in altri Stati ad ordinamento regionale, che richiamavano la necessità di inserire la soluzione del "caso" italiano in un più ampio dibattitto culturale sull'Europa e le sue regioni.

\section{Bibliografia}

Aimo P. 2007, Le Province nel regime fascista, "Storia Amministrazione Costituzione", 15

Aimo P. (ed.) 2009, Le Province dalle origini alla Costituzione, Milano: Isap

Anderlini F. 2012, Alleanze sociali e rapporti politici nel "modello emiliano" storico. I mutamenti dell'ultimo quarto di secolo, in De Maria C. (ed.) 2012, Bologna futuro. Il "modello emiliano" alla sfida del XXI secolo, Bologna: Clueb

Balzani R. 2004, Dal "modello emiliano" alla Regione Emilia Romagna, in Neri Serneri S. (ed.) 2004, Alle origini del governo regionale. Culture, istituzioni, politiche in Toscana, Roma: Carocci

Barbagallo F. 2003, Il Pci dal sequestro di Moro alla morte di Berlinguer, in De Rosa G. e Monina G. (eds.) 2003, L'Italia repubblicana nella crisi degli anni Settanta. Sistema politico e istituzioni, Soveria Mannelli: Rubbettino

Barbera A. 1980, Sintesi della ricerca sul riordino istituzionale della Regione, "Regione e governo locale", 1

Bianchi G. 1986, «Maturità precoce»: una modernizzazione a rischio, in Mori G. (ed.) 1986, Storia d'Italia. Le Regioni dall'Unità a oggi. La Toscana, Torino: Einaudi 
Bianchi P. 1987, Emilia Romagna. Problemi e prospettive, in Finzi R. (ed.) 1987, Storia d'Italia. Le regioni dall'Unità ad oggi. L'Emilia Romagna, Torino: Einaudi

Bin R. 1982, Leggi regionali per la programmazione, "Regione e governo locale”, 1

Bonora P. 2005, Orfana e claudicante. L'Emilia “post-comunista” e l'eclissi del modello territoriale, Bologna: Baskerville

Bortolotti L. 1986, L'evoluzione del territorio, in Mori G. (ed.) 1986, Storia d'Italia. Le Regioni dall'Unità a oggi. La Toscana, Torino: Einaudi

Bottini F. 2003, Sovracomunalità. 1925-1970, Milano: FrancoAngeli

Cammelli M. 1978, Politica istituzionale e modello emiliano: ipotesi per una ricerca, "Il Mulino", 259

Cammelli M. 1980, Regione e poteri locali oggi: premesse e modalità di un rapporto attuale, "Regione e governo locale", 1

Campos Venuti G. 2010, L'urbanista e l'amministratore. Dialogo con Giuseppe Campos Venuti, in Boarelli M., Lambertini L. e Perrotta M. (eds.) 2010, Bologna al bivio. Una città come le altre?, Roma: Edizioni dell'Asino

Cassese S. 1986, Centro e periferie in Italia. I grandi tornanti della loro storia, "Rivista trimestrale di diritto pubblico", 2

Cortese M. 1981, L'esperienza dei comprensori nel Veneto, "Regione e governo locale", 1

Crainz G. 2009, Autobiografia di una repubblica. Le radici dell'Italia attuale, Roma: Donzelli

De Marchi G. 1981, Pianificazione territoriale comprensoriale, "Regione e governo locale", 5

De Maria C. 2009, Marcello Stefanini, il Comune e le autonomie locali, in Ridolfi M. (ed.) 2009, Il Comune democratico. Autogoverno, territorio e politica a Pesaro negli anni di Marcello Stefanini (1965-1978), Milano: FrancoAngeli

De Maria C. 2011a, Il “modello emiliano” nella storia d'Italia. Appunti di lavoro, “Officina della Storia”, 6

De Maria C. 2011b, L'evoluzione del Terzo settore dal Novecento a oggi (1915-2011), in Rossi E. e Zamagni S. (eds.) 2011, Il Terzo settore nell'Italia unita, Bologna: il Mulino

De Maria C. 2012, Il "modello emiliano”: una prospettiva storica, in De Maria C. (ed.) 2012, Bologna futuro. Il “modello emiliano” alla sfida del XXI secolo, Bologna: Clueb

De Siervo U. 2003, La difficile attuazione delle regioni, in De Rosa G. e Monina G. (eds.) 2003, L'Italia repubblicana nella crisi degli anni Settanta. Sistema politico e istituzio$n i$, Soveria Mannelli: Rubbettino

Emiliani G. 1981, I provvedimenti sulla finanza locale del 1980-81. Una occasione perduta?, "Regione e governo locale", 2

Fanti G. 1970, La politica delle alleanze in una "Regione rossa", "Critica marxista", 3

Fanti G. 1993, Anni Settanta: le ragioni di una scelta, "Ibc", 5 
Fanti G. e Ferri G.C. 2001, Cronache dall'Emilia rossa. L'impossibile riformismo del Pci, prefazione di L. Pedrazzi, Bologna: Pendragon

Farneti P. 1983, Il sistema dei partiti in Italia. 1946-1979, Bologna: il Mulino

Fioravanti M. 2003, La trasformazione del modello costituzionale, in De Rosa G. e Monina G. (eds.) 2003, L'Italia repubblicana nella crisi degli anni Settanta. Sistema politico e istituzioni, Soveria Mannelli: Rubbettino

Fofi G. 1994, Introduzione, in Marcon G. e Nonno M. (eds.) 1994, Il paese nascosto. Storie di volontariato, Roma: E/O

Ingrao P. 1973, Novità dalle Regioni, "Rinascita”, 19

Istat 2011, L'Italia in 150 anni. Sommario di statistiche storiche 1861-2010, Roma: Istat

Istituto regionale programmazione Toscana 1979, La questione comprensoriale e l'individuazione di aree funzionali in Toscana, Firenze

Lambertini L. 2012, I servizi socio-sanitari ed educativi. Storie da ricostruire per cercare risposte alla crisi di oggi, in De Maria C. (ed.) 2012, Bologna futuro. Il "modello emiliano" alla sfida del XXI secolo, Bologna: Clueb

Martinelli C. 1983, Le entrate tributarie dei Comuni e delle Province, "Regione e governo locale", 1

Massari O. 1987, Le elezioni regionali nella dinamica centro-periferia: un voto ambiguo, in Caciagli M. e Corbetta P. (eds.) 1987, Elezioni regionali e sistema politico nazionale. Italia, Spagna e Repubblica federale tedesca, Bologna: il Mulino

Onida V. 1981, Un passo avanti sulla via della riforma della finanza locale, "Regione e governo locale", 2

Passigli S. 1987, Introduzione, in Morisi M. (ed.) 1987, Regioni e rappresentanza politica. Questioni e materiali di ricerca sui Consigli regionali, Milano: FrancoAngeli

Pastori G. 1980, Le regioni senza regionalismo, "Il Mulino", 2

Pci, Comitato regionale Emilia Romagna 1967, Linee per una politica di programmazione dello sviluppo economico e sociale in Emilia Romagna, in D'Attorre P.P. (ed.) 1981, I comunisti in Emilia Romagna. Documenti e materiali, Bologna: Istituto Gramsci

Pci, Comitato regionale Emilia Romagna 1973, La riforma democratica dello Stato. Lo Stato delle regioni e delle autonomie locali. Le deleghe della regione ai comuni e alle province, Imola: Galeati

Polsi A. 2004, Profilo dell'ente Provincia dall'unificazione al fascismo, "Storia Amministrazione Costituzione", 12

Regione Emilia Romagna 1982, Quadro di riferimento del programma regionale di sviluppo 1982-1985, "Regione Emilia Romagna. Bollettino ufficiale", 29 luglio

Ridolfi M. 2010, Storia politica dell'Italia repubblicana, Milano: Bruno Mondadori

Rotelli E. 1991, Il martello e l'incudine. Comuni e Province fra cittadini e apparati, Bologna: il Mulino

Sabbatucci G. 2003, I socialisti e la solidarietà nazionale, in De Rosa G. e Monina G. 
(eds.) 2003, L'Italia repubblicana nella crisi degli anni Settanta. Sistema politico e istituzioni, Soveria Mannelli: Rubbettino

Scoppola P. 2003, Una crisi politica e istituzionale, in De Rosa G. e Monina G. (eds.) 2003, L'Italia repubblicana nella crisi degli anni Settanta. Sistema politico e istituzio$n i$, Soveria Mannelli: Rubbettino

Tarrow S. 1979a, Tra centro e periferia. Il ruolo degli amministratori locali in Italia e in Francia, Bologna: il Mulino

Tarrow S. 1979b, Decentramento incompiuto o centralismo restaurato? L'esperienza regionalistica in Italia e in Francia, "Rivista italiana di scienza politica", 2

Urbani P. 1981, Pianificazione territoriale regionale e comprensoriale, "Regione e governo locale", 5

Vandelli L. 1982, I modelli associativi nei progetti di riforma del governo locale, "Regione e governo locale", 2

Vandelli L. 1990, Poteri locali. Le origini nella Francia rivoluzionaria. Le prospettive nell'Europa delle regioni, Bologna: il Mulino

Vaudagna M. 1983, Fallito il modello torinese, vince l'emiliano. La Germania se ne accorge, l'Italia no. Intervista a Charles Sabel del Massachussetts Institute of Technology, "Il Manifesto", 13 maggio

Zangheri R. 1975, Decentramento e partecipazione democratica, in D’Attorre P.P. (ed.) 1981, I comunisti in Emilia Romagna. Documenti e materiali, Bologna: Istituto Gramsci

Zangheri R. 1978, I Comuni, in Programmazione autonomie partecipazione. Un nuovo ordinamento dei poteri locali. Atti del convegno di studi promosso dal Centro studi e iniziative per la riforma dello Stato e dall'Istituto Gramsci, Roma, 23-25 gennaio 1978, vol. I, Roma: Edizioni delle autonomie

\section{Risorse}

Archivio storico della Regione Emilia Romagna:

http://www.self-pa.net/CorsiPubblici/archivio/index.htm

Biblioteca dell'Assemblea legislativa della Regione Emilia Romagna: http://www.assemblea.emr.it/biblioteca/

La Regione e la sua storia: http://lanostrastoria.regione.emilia-romagna.it/flex/cm/pages/ServeBLOB.php/L/IT/ IDPagina/100

Archivi della Fondazione Gramsci Emilia Romagna: http://www.iger.org/archivio/i-fondi/ 